\title{
Hadassah \\ Cardiac Arrest Following Reversal of Muscle Relaxation by Neostigmine: A Case Report
}

\author{
Tural Alekberli MD, M. Al Barade M.B.,B.CH, A. Berkowitz MD, Y. Plotkin MD, G. Landesberg MD
}

Hadassah Hebrew University Medical Center, Department of Anesthesiology and CCM - Jerusalem (Israel)

Background: Acetylcholinesterase inhibiting (AChE-I) drugs are typically administered to facilitate the recovery from the neuromuscular blockade caused by nondepolarizing neuromuscular blocking drugs(NMBD), at the end of surgical procedures and before extubation. AChE-I, including neostigmine, block acetylcholinesterase (AChE) at all peripheral cholinergic synapses and therefore have significant parasympathomimetic activity normally attenuated, or abolished, by the co-administration of an antimuscarinic agent, e.g. atropine or glycopyrrolate. However, potentially fatal complications, such as severe bradycardia and cardiac arrest, may still occur [1-4]. We report a case of cardiac arrest, requiring full cardiopulmonary resuscitation (CPR) during emergence from general anesthesia, in a patient with borderline 1st degree atrioventricular (AV) block and incomplete right bundle-branch block (ICRBBB) following neostigmine administration.

Case Report: 82-year-old male patient, weighing $65 \mathrm{~kg}$, with a background of type 2 diabetes mellitus, dyslipidemia, iron-deficiency anemia, hiatal hernia, lumbar spinal stenosis, cataract and peptic ulcer disease underwent urgent repair of an incarcerated inguinal hernia. Diagnostic percutaneous transluminal coronary angioplasty (PTCA) 12 years earlier was normal. No other cardiac intervention was performed and the patient denied cardiovascular diseases. Although medications (Oral antidiabetic agent, iron supplementation and statin) were prescribed for the patient at home, he was not using any of those medications. His preoperative ECG revealed borderline 1st degree AV block (PR interval $210 \mathrm{msec}$ ) with incomplete right bundlebranch block (ICRBBB) (HR-66 BPM) (Figure 1). His preoperative serum potassium was $4.5 \mathrm{mmol} / \mathrm{l}$, serum hemoglobin concentration was $9.8 \mathrm{Gr} \%$, and serum glucose was $6.7 \mathrm{mmol} / \mathrm{l}$. The rest of the routine blood tests were within normal ranges. He had normal vital signs before induction of anesthesia. Rapid-sequence induction was performed with etomidate $20 \mathrm{mg}$ and succinylcholine $70 \mathrm{mg}$. After induction of anesthesia intra-arterial line was inserted because of the old age of the patient, the urgent operation and the poor medical history. The other intraoperative medications included IV fentanyl $100 \mathrm{mg}$, morphine $4 \mathrm{mg}$, dexamethasone $4 \mathrm{mg}$, ondansetron $6 \mathrm{mg}$, antibiotics and rocuronium $20 \mathrm{mg}$. Anesthesia was maintained using isoflurane. Surgery was uneventful; the patient required no blood products or vasopressor agents during approximately 2 hours of the procedure. Isoflurane administration was stopped at a timely fashion and the patient exhibited signs of spontaneous emergence from neuromuscular blockade; neostigmine $0.04 \mathrm{mg} / \mathrm{kg}(2.5 \mathrm{mg})$ with atropine $0.15 \mathrm{mg} / \mathrm{kg}$ (1 $\mathrm{mg}$ ) (both diluted to $10 \mathrm{ml}$ with NS) were given. Shortly $(30 \mathrm{sec})$ thereafter, cardiac arrest was noted on the ECG and invasive blood pressure (IBP) monitor. CPR with chest compressions was started immediately and continued for approximately 4 minutes to include also the administration of IV atropine $1 \mathrm{mg}$, IV adrenaline 1 $\mathrm{mg}$, and IV calcium chloride $1 \mathrm{~g}$ (due to high serum potassium level). On urgent laboratory tests during CPR, serum potassium was 9.54 $\mathrm{mmol} / \mathrm{l}$, which returned to normal $(4.47 \mathrm{mmol} / \mathrm{l})$ only after return of spontaneous circulation (ROSC). The patient remained hemodynamically unstable and required a noradrenaline infusion (0.1 $\mathrm{mcg} / \mathrm{kg} / \mathrm{min}$ ) through an internal jugular central venous catheter that was inserted following the CPR. IV rocuronium $20 \mathrm{mg}$ was readministered to facilitate ventilating the patient properly during transport to the intensive care unit (ICU). The patient was extubated on his 1st day in the ICU and was discharged to the surgical ward after one day. He was discharged home after complete recovery on postoperative day 10 after obtaining a cardiological consultation and transthoracic echocardiography which showed upper limits of normal diameter of LV, mildly reduced global systolic function, EF- $40 \%$, basal septal "knuckle" with no LVOT obstruction. Moderately dilated LA and RA. Normal size and normal global systolic function of RV. Without significant valvular disease.
Figure 1:

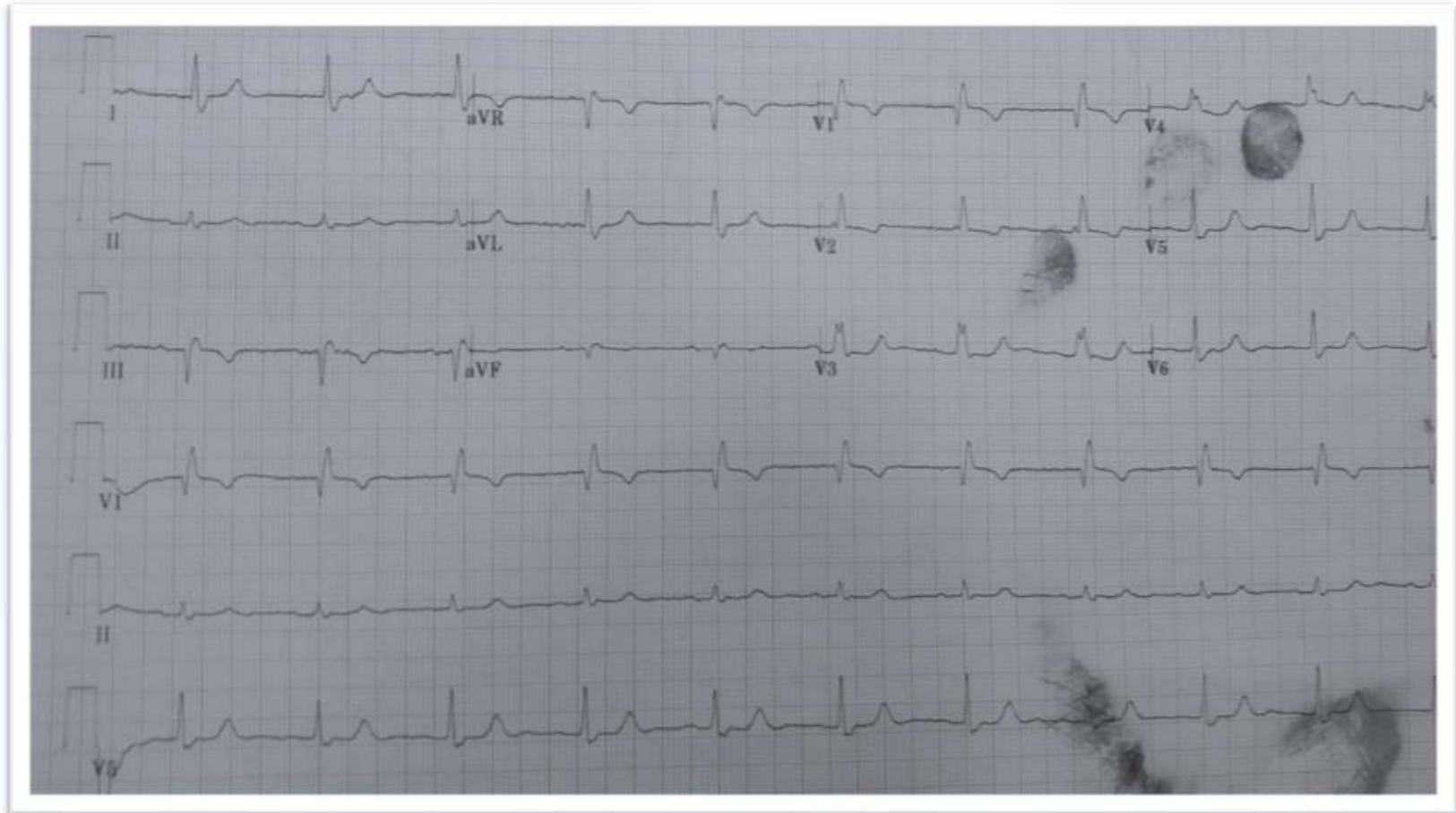

Borderline $1^{\text {st }}$ degree AV block (PR-interval 210msec) with ICRBBB, HR-66 BPM

Discussion and Learning Points: Neostigmine is a potent anticholinergic agent. Nevertheless, complete cardiac arrest following co-administration of neostigmine with a cholinergic agent drug like atropine is very rare. Cardiac complications such as QT interval prolongation or even ST elevation and takotsubo cardiomyopathy related to AChE-I such as neostigmine and pyridostigmine were reported, particularly in patients with myasthenia gravis or Brugada syndrome [7-9]. Additionally, cardiac arrests following neostigmine administration were reported in a case of combined general and spinal anesthesia, in pediatric or cardiac transplant patients [8] and in a patient with a background of coronary vasospasm[9] undergoing noncardiac surgery. To the best of our knowledge, cardiac arrest requiring CPR for 4 minutes after the administration of a single dose of neostigmine has not been previously reported. The prevalence of preoperative borderline 1st- degree AV block and ICRBBB as were seen in our patients is relatively common and we do not know to what extent they contributed to the cardiac arrest. We wish to emphasize therefore that the use of neostigmine for reversal of muscle paralysis may be dangerous. Perioperative neuromuscular monitoring to avoid unnecessary use of NMDBs and AChE-I or the alternative use of sugammadex [10] may prevent this complication. Elderly patients, especially those with conduction abnormalities on preoperative ECG or significant coronary disease are at particular risk.

References:

1. Kido K, Mizuta K, Mizuta F, Yasuda M, Igari T, Takahashi M. Coronary vasospasm during the reversal of neuromuscular block using neostigmine. Acta Anaesthesiol Scand (2005); 49: 1395-6.

2. Suzuki M, Yoshii T, Ohtsuka T, Sasaki O, Hara Y, Okura T, et al Coronary spastic angina induced by anticholinesterase medication for myasthenia gravis a case report. Angiology (2000); 51: 1031-4.

3. Tüfek A, Yildirim B, Tokgöz O, Karaman H, Çelik F, Aycan IÖ. Immediate cardiac arrest after neostigmine administration. J Pak Med Assoc (2012); 62: 609.

4. Yamabe H, Yasue H, Okumura K, Ogawa H, Obata K, Oshima S. Coronary spastic angina precipitated by the administration of an anticholinesterase drug (distigmine bromide). Am Heart J (1990);120:211-3.

5. Cardiac Arrest Following the Administration of Neostigmine J. I. LAWSON Brit. J. Anaesth. (1956), 28, 336

6. Nkemngu NJ. Asystole following neuromuscular blockade reversal in cardiac transplant patients .Ann Card Anaesth (2017);20:385-6.

7. Cardiac arrest following reversal of muscle relaxation by pyridostigmine -A case report - Jun Young Jo, Jung Min Yi, Yoon Kyung Lee*, Seung Woo Ku, and Pyung Hwan Park. Anesth Pain Med (2014); 9: 205-208

8. Battineni A, Mullaguri N, Thanki S, Chockalingam A, Govindarajan R. A Case Report of Recurrent Takotsubo Cardiomyopathy in a Patient during Myasthenia Crisis. Case Rep Crit Care. 2017;2017:5702075. [Epub 2017 Oct 19].

9. Tsutsumi YM, Tomiyama Y, Horikawa YT, Sakai Y, Ohshita N, Tanaka K, Oshita S. General anesthesia for electroconvulsive therapy with Brugada electrocardiograph pattern. J Med Invest. 2011 Aug;58(3-4):273-6.

10. Plaud, B., et al. "Efficacy, Safety, and Pharmacokinetics of Sugammadex for the Reversal of Rocuronium-induced Neuromuscular Blockade in Elderly Patients (vol 114, pg 318, 2011)." ANESTHESIOLOGY 127.3 (2017): 591-591. 